\title{
Bimetallic CoPt nanoparticles-modified Pt disk electrode for the amperometric sensing of ascorbic acid
}

\author{
MERZAK DOULACHE ${ }^{\mathrm{a}, \mathrm{b}, *}$, MOHAMED TRARI ${ }^{\mathrm{b}}$ and BOUBAKEUR SAIDAT ${ }^{\mathrm{a}}$ \\ a Laboratory of Physical Chemistry of Materials (LPCM), Faculty of Sciences, (UATL), BP 37G, \\ 03000 Laghouat, Algeria \\ b Laboratory of Storage and Valorization of Renewable Energies (LSVRE), Faculty of Chemistry, (USTHB), \\ BP 32, El Alia, 16111 Algiers, Algeria \\ E-mail: doulache@yahoo.fr
}

MS received 5 April 2018; revised 25 May 2018; accepted 8 June 2018; published online 2 August 2018

\begin{abstract}
Cobalt-Platinum bimetal nanoparticles (CoPtNPs) were electrodeposited on bare Pt electrode. A highly sensitive amperometric sensor-based on CoPtNPs/Pt modified electrode was constructed to determine ascorbic acid (AA). The Co-Pt nanoparticles were characterized by scanning electron microscopy (SEM) and cyclic voltammetry $(\mathrm{CV})$. Due to the synergistic effects of Co-Pt nanoparticles, the electrocatalytic activity of modified electrode showed a significant enhancement toward the oxidation of AA compared to Co/Pt and bare Pt electrodes in alkaline medium. The electrocatalytic activity of CoPtNPs/Pt was studied as a function of Co:Pt molar ratio in the deposition bath. The analytical performance of the proposed sensor has been investigated by chronoamperometry method under the optimized conditions. This sensor showed a linear response to AA concentration range from $10 \mu \mathrm{M}$ to $0.5 \mathrm{mM}$ with a detection limit of $0.37 \mu \mathrm{M}(\mathrm{S} / \mathrm{N}=3)$, a high sensitivity of $103 \mu \mathrm{A} \mathrm{mM}^{-1}$, and a fast response time. In addition, the reproducibility, selectivity and applicability of this sensor were assessed with satisfactory results.
\end{abstract}

Keywords. Co-Pt bimetallic nanoparticles; modified electrode; electrocatalysis; ascorbic acid; electrochemical sensor.

\section{Introduction}

Ascorbic acid (AA), known as Vitamin C, is one of the important vitamins found in fruits and vegetables, which help to maintain normal physiological functions. AA, widely known as antioxidant, also plays a crucial role in the metabolism of cholesterol. The important role of AA has been revealed through recent researches in various fields such as toxicology, genetics, oncology, nutritional science, and even material science as a mild reducing agent. ${ }^{1}$ The toxicity of reactive oxygen species ${ }^{2}$ and synthetic pyrethroid pesticide ${ }^{3}$ can be protected by AA reducing toxic substances. Hence, the development of a simple and reliable method for the determination of AA with a high sensitivity and a good selectivity is of great importance. Among the reported methods, the electrochemical method is considered to be a crucial topic of interest due to its simplicity and rapidity without the sample preparation procedures. ${ }^{4-13}$

\footnotetext{
*For correspondence
}

However, the direct oxidation of AA at bare electrodes is irreversible and the subsequent hydrolysis product, 2,3-diketogluconic acid ${ }^{14}$ is readily adsorbed onto the electrodes surface. Moreover, some of biological molecules, like the dopamine and uric acid are oxidized at almost the same potential at conventional electrodes and results in an overlap of voltammetric response. Therefore, using bimetallic nanoparticles modified electrodes has been described as promising route for the electrochemical AA sensor fabrication. ${ }^{15-19}$ It has been reported that bimetallic nanoparticles such as PdNi, AuPt, AuAg, AgPt and PtNi, exhibit better electrochemical properties than their single-metal component. ${ }^{19-23}$ Specifically, bimetallic CoPt NPs are believed to offer unprecedented benefits in catalysis by tuning the catalysts durability. ${ }^{24}$

In order to get a better electrochemical sensor with high sensitivity, selectivity and stability, we have investigated the electrocatalytic activity of CoPt-based bimetallic nanomaterials. This kind of electrodes can interact synergistically to form redox mediators with improved activity attributed to a larger surface area, 
excellent catalytic activity, enhanced conductivity and good stability. ${ }^{25,26}$

Aiming to achieve a high catalytic effect with increased stability and lower cost, we dispersed $\mathrm{CoPt}$ nanoparticles on bare Pt disk electrode to produce $\mathrm{CoPt} / \mathrm{Pt}$ nanomaterial. In this, contribution, the electrochemical activity of the CoPt/Pt modified Pt electrode for AA oxidation was studied and compared with $\mathrm{Co} / \mathrm{Pt}$ and bare Pt electrodes. The results indicate enhanced electrocatalytic activity of CoPtNPs/Pt and a higher sensitivity, compared to $\mathrm{Co} / \mathrm{Pt}$ modified electrode ${ }^{27}$ and bare Pt electrode toward the AA oxidation. This study demonstrated that $\mathrm{CoPt} / \mathrm{Pt}$ nanomaterial is a promising catalyst for amperometric sensing of ascorbic acid.

\section{Experimental}

\subsection{Reagents and apparatus}

Chloroplatinic acid hexahydrate $\mathrm{H}_{2} \mathrm{PtCl}_{6}$ (99\%, Merck, Germany) and $\mathrm{CoSO}_{4}(>99 \%$, Merck, Germany) were used in the preparation of modified electrode. Ascorbic acid (99.5\%), $\mathrm{H}_{3} \mathrm{BO}_{3}(99.5 \%)$ and $\mathrm{NaOH}(99 \%)$ were analytical grade products from Fluka. Deionized water was used in the preparation of solutions.

The electrochemical experiments were realized with a PGZ402 potentiostat/galvanostat (Radiometer analytical). A platinum foil and saturated calomel electrode (SCE) were used as a counter and reference electrode, respectively, in a standard three-electrode cell; the working electrode was Pt disk electrode $(\varnothing=2 \mathrm{~mm})$. For steady-state amperometric experiments, the working potential was fixed at $0.2 \mathrm{~V}$ and the solution was stirred gently with a magnetic stirrer. Every test was repeated three times in order to confirm the reproducibility of our results. The surface morphology of CoPtNPs/Pt electrode was analyzed with a JOEL JSM 6360-LV microscope.

\subsection{Preparation of CoPtNPs/Pt}

Before each experiment, the working electrode was handpolished in aqueous suspensions of successively finer grades of alumina (5 to $0.3 \mu \mathrm{m}$ ), until a mirror-finishing surface was obtained. Then, it was ultrasonically cleaned for $5 \mathrm{~min}$ in the mixture $\mathrm{HNO}_{3}$ /absolute ethanol (1/1), thoroughly rinsed with water and dried at room temperature. CoPt NPs were first deposited electrochemically on the bare Pt electrode surface by cathodic reduction of a mixed solution containing $\mathrm{CoSO}_{4}, \mathrm{H}_{2} \mathrm{PtCl}_{6}$ and $\mathrm{H}_{3} \mathrm{BO}_{3}(0.05 \mathrm{M})$ during 120 s at a potential of $-1.2 \mathrm{~V}$. Various molar ratios of cobalt and platinum were employed. The total concentrations $\mathrm{CoSO}_{4}$ and $\mathrm{H}_{2} \mathrm{PtCl}_{6}$ were $0.03 \mathrm{M}$ with Co:Pt molar ratios of 2:1, 3:1, 4:1 and 5:1. After rinsing with distilled water, the modified electrode was immersed in an alkaline solution $(\mathrm{NaOH} 0.1 \mathrm{M})$ and scanned repetitively for 20 cycles in the potential range $(-0.6$ to 0.6
V) at a rate of $50 \mathrm{mV} \mathrm{s}^{-1}$ until a stable cyclic voltammogram (CV) was reached.

\subsection{Preparation of real samples}

Pharmaceutical tablets of Vitamin C (UPSA ${ }^{\circledR} 500 \mathrm{mg}$, Saidal Company, Algiers) were tested for the ascorbic acid. The tablets were dissolved in $\mathrm{NaOH}$ solution $(0.1 \mathrm{M})$ and diluted to prepare solutions of 50,100 and $250 \mu \mathrm{M}$. The validity of $\mathrm{CoPtNPs} / \mathrm{Pt}$ modified electrode for the AA determination was assessed by direct determination using a previously calibrated graph. All solutions were prepared using ultra-high-quality water (Millipore).

\section{Results and Discussion}

\subsection{Electrochemical deposition of bimetallic cobalt-platinum}

The electrochemical behavior of $\mathrm{Co}^{2+}, \mathrm{Pt}^{4+}$ and $\mathrm{Co}-\mathrm{Pt}$ binary electrolyte was studied by cyclic voltammetry method in $0.05 \mathrm{M} \mathrm{H}_{3} \mathrm{BO}_{3}$ (pH 5.25). The $\mathrm{CV}$ in an electrolyte containing $\mathrm{Co}^{2+}$ ions (Figure 1a) shows a huge rise in cathodic current at $-1.1 \mathrm{~V}$ (peak $\mathrm{I}_{\mathrm{c}}$ ) which corresponds to the reduction of $\mathrm{Co}^{2+}$ and its corresponding oxidation peak $\left(\mathrm{I}_{\mathrm{a}}\right)$ appears at $0.27 \mathrm{~V}$ during the anodic sweep. At a potential higher than $-1.35 \mathrm{~V}$, hydrogen evolution takes place with increased current.

$\mathrm{CV}$ obtained in $\mathrm{Pt}^{4+}$ electrolyte (Figure $1 \mathrm{~b}$ ) shows two overlapping reduction waves at $0.18 \mathrm{~V}\left(\mathrm{I}_{\mathrm{c}}\right)$ and -0.09 $\mathrm{V}\left(\mathrm{II}_{\mathrm{c}}\right)$ correspond to the reduction of $\mathrm{PtCl}_{6}^{2-}$ to $\mathrm{PtCl}_{4}^{2-}$ and $\mathrm{PtCl}_{4}^{2-}$ to $\mathrm{Pt}$ respectively, which can be expressed as: ${ }^{28,29}$

$\mathrm{PtCl}_{6}^{2-}+2 \mathrm{e}^{-} \rightarrow \mathrm{PtCl}_{4}^{2-}+2 \mathrm{Cl}^{-}$
$\mathrm{PtCl}_{4}^{2-}+2 \mathrm{e}^{-} \rightarrow \mathrm{Pt}+4 \mathrm{Cl}^{-}$

In the cathodic region, the $\mathrm{CV}$ curves showed the adsorption/desorption peaks of atomic hydrogen $\left(\mathrm{III}_{\mathrm{c}}\right.$ and $\left.\mathrm{IV}_{\mathrm{c}}\right)$, characteristic of the bulk Pt electrode around -0.29 to $-0.69 \mathrm{~V}$, suggesting an electrodeposition of Pt particles on the electrode.

$\mathrm{CV}$ in Co-Pt binary electrolyte shows three reduction peaks assigned to the reduction of cobalt $\left(\mathrm{I}_{\mathrm{c}}\right)$ and $\mathrm{Pt}^{4+}$ ions $\left(\mathrm{II}_{\mathrm{c}}, \mathrm{III}_{\mathrm{c}}\right)$ by comparison with the peaks obtained for individual metal ion electrolytes (Figure 1c). The $\mathrm{Co}^{2+}$ reduction potential is slightly shifted toward positive potentials, which could be attributed to the alloy formation. The peaks obtained during the anodic scan can be attributed to the oxidation of cobalt $\left(\mathrm{I}_{\mathrm{a}}\right)$ and for Co-Pt alloy $\left(\mathrm{II}_{\mathrm{a}}\right)$. It is obvious from the above discussion that to achieve a codeposition of cobalt and platinum on bare Pt electrode, a minimal potential of $-1 \mathrm{~V}$ is required. 

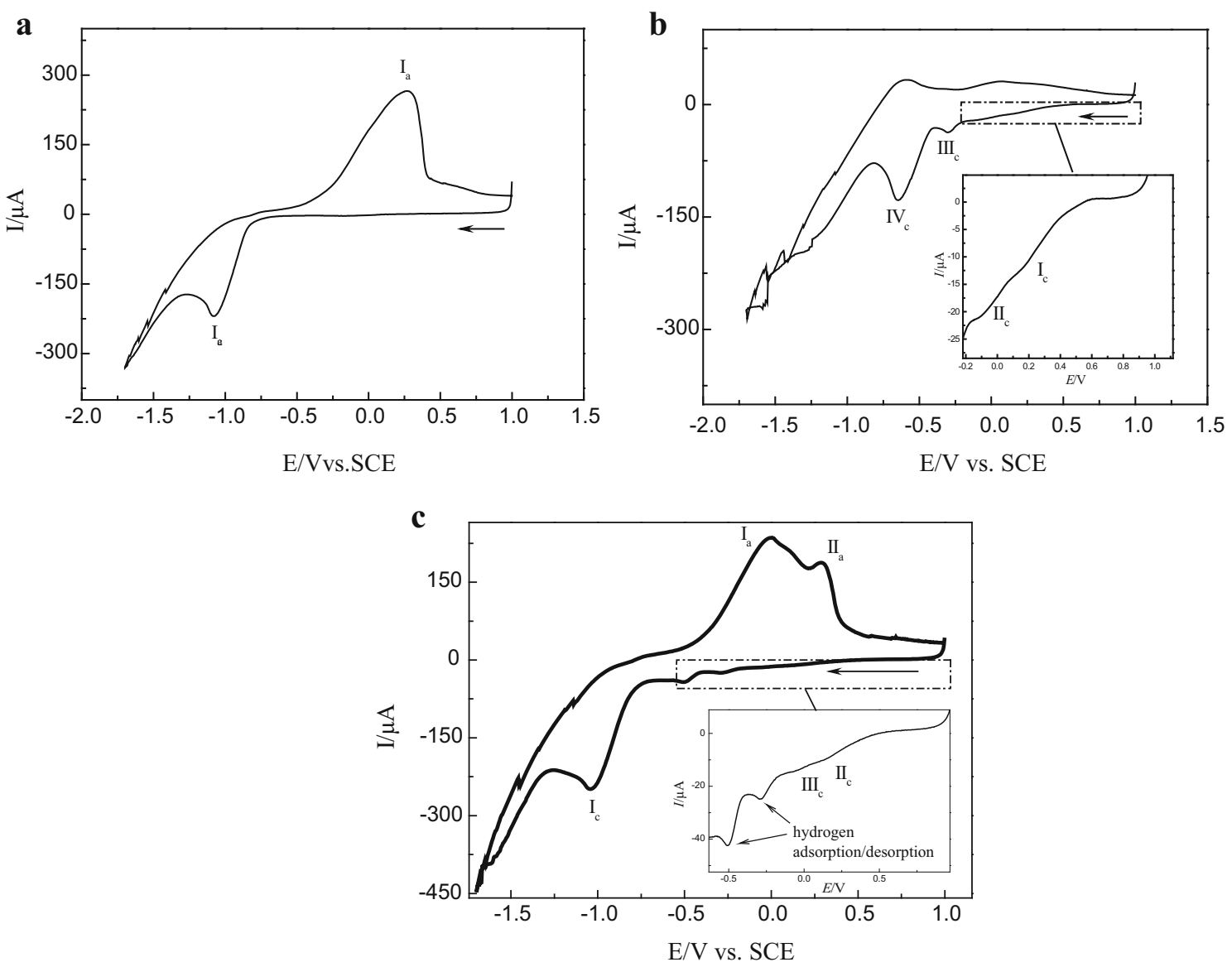

Figure 1. $\mathrm{CVs}$ recorded on bare Pt electrode at a scan rate of $50 \mathrm{mV} \mathrm{s}^{-1}$ in an electrolyte of $0.05 \mathrm{M} \mathrm{H}_{3} \mathrm{BO}_{3}$ containing (a) $0.02 \mathrm{M} \mathrm{CoSO}_{4}$, (b) $0.01 \mathrm{M} \mathrm{H}_{2} \mathrm{PtCl}_{6}$ and (c) $0.02 \mathrm{M} \mathrm{CoSO}_{4}+0.01 \mathrm{M} \mathrm{H}_{2} \mathrm{PtCl}_{6}$ at a pH 5.25.

Hence, for all deposition studies, a potential of $-1.2 \mathrm{~V}$ is used.

After electrodeposition, the CoPt bimetallic film was conditioned in $0.10 \mathrm{M} \mathrm{NaOH}$ solution by potential cycling between -0.6 and $0.6 \mathrm{~V}$ with 20 cycles $\left(50 \mathrm{mV} \mathrm{s}^{-1}\right)$. These parameters were obtained experimentally as optimum values for development of similar quantities of Co redox mediators, and the maximum activation of the electrode surface for the electrocatalytic oxidation of AA.

\subsection{Scanning electron microscopy characterization (SEM)}

The SEM analysis is performed in order to define the morphological properties of the Co-Pt deposit (4:1 molar ratio). In this respect, we have compared the SEM micrograph of CoPt with Co particles deposited individually under similar electrochemical pulsed conditions.

Figure 2b shows SEM micrograph of Co-Pt on Pt disk electrodes. The particles of Co and Pt covering the whole electrode surface with irregular sizes lying between 50 and $100 \mathrm{~nm}$. The individual Co deposit on the Pt surface (Figure 2a) shows that the Co particles are formed of nearly spherical structures dispersed uniformly with well-defined geometric shapes. The mean grains size of single Co particles lies between 300 and $800 \mathrm{~nm}$. So, the difference between the CoPt systems and single deposits of Co indicates that the morphological properties of the binary deposit are mutually and markedly influenced by the existence of individual metals. The CoPt deposit appears formed of resolved and well-dispersed single particles with a higher surface roughness. The surface distribution of CoPt NPs deposit affects the real surface area, thus generating the increased active surface of the exposed particles and in this way, more active sites are available in comparison with individual Co deposit. This may make the roughened CoPtNPs/Pt electrode an efficient material for the electron transfer with a greater catalytic activity for the AA electrooxidation.

\subsection{Characterization of CoPt NPs/Pt electrode}

The $20^{\text {th }}$ cyclic voltammograms $(\mathrm{CVs})$ of $\mathrm{Co} / \mathrm{Pt}$ and CoPtNPs/Pt electrodes (with different Co:Pt molar 

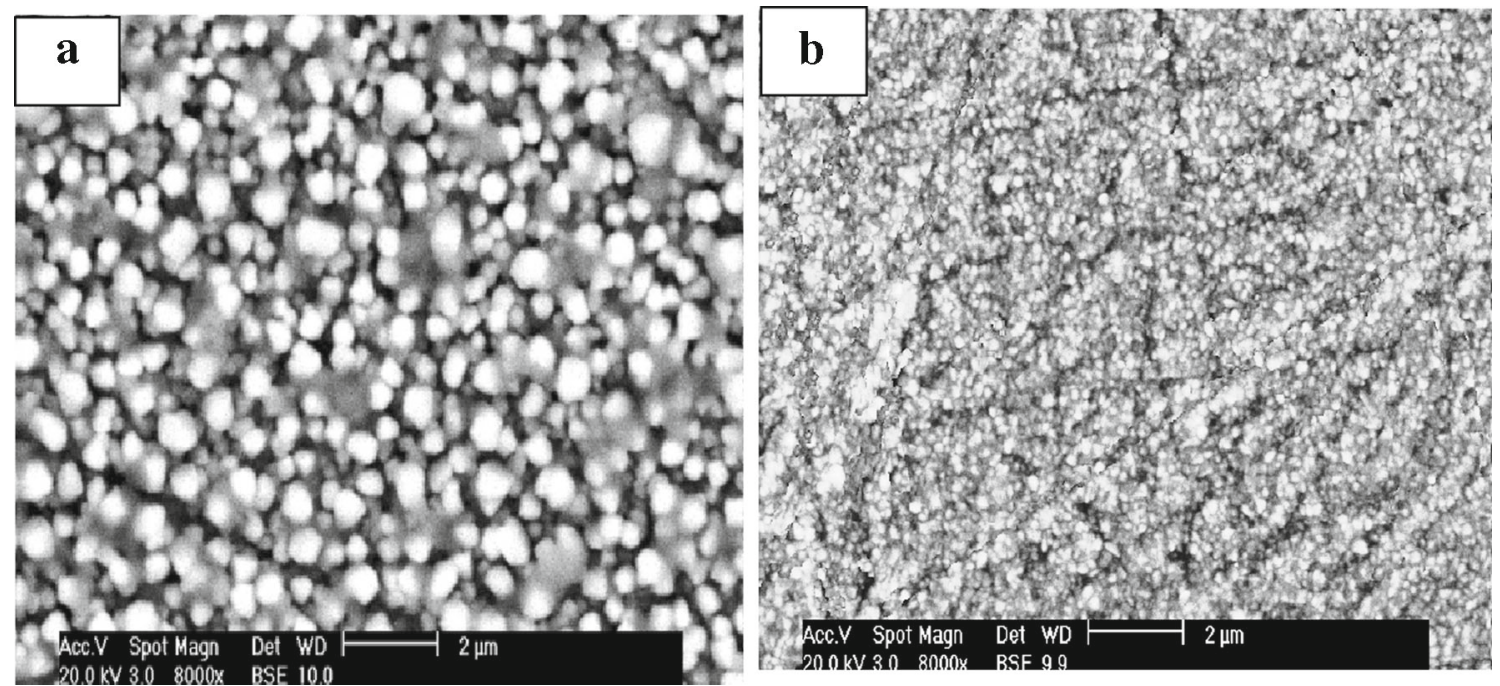

Figure 2. The SEM image of (a) Co/Pt surface electrode and (b) CoPtNPs/Pt (the Co:Pt molar ratio is 4:1) surface electrode.

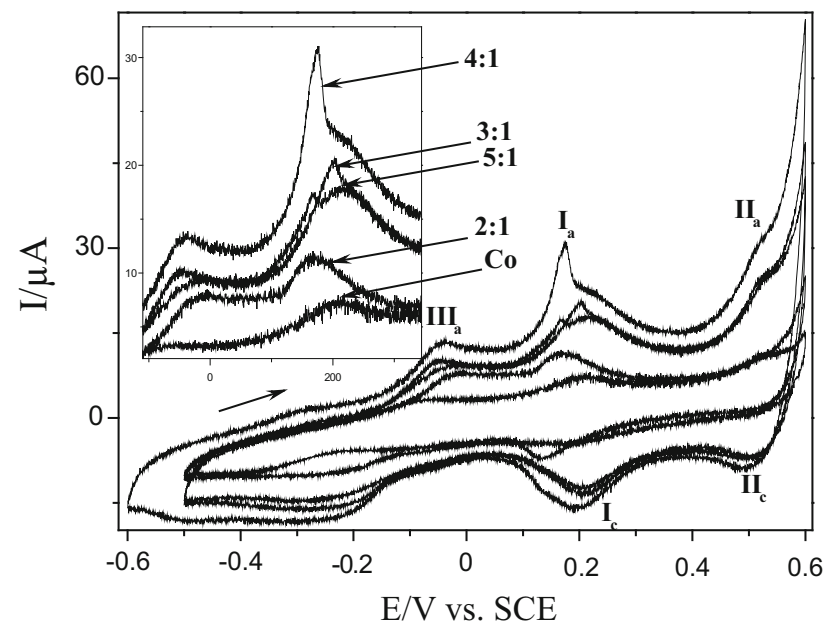

Figure 3. $\mathrm{CVs}$ of $\mathrm{Co} / \mathrm{Pt}$ electrode and $\mathrm{CoPtNPs} / \mathrm{Pt}$ electrodes (with different Co:Pt molar ratios 2:1, 3:1, 4:1 and $5: 1)$ in $0.1 \mathrm{M} \mathrm{NaOH}$ at a scan rate of $50 \mathrm{mV} \mathrm{s}^{-1}$. Inset shows the zoom view of the peak currents.

ratios) in $0.1 \mathrm{M} \mathrm{NaOH}$ solution are illustrated in Figure 3. The electrochemical behavior of CoPtNPs/Pt electrodes looks like that of $\mathrm{Co} / \mathrm{Pt}$ where the anodiccathodic peaks provide evidence of $\mathrm{Co}^{\mathrm{III}} / \mathrm{Co}^{\mathrm{II}}$ (peak $\mathrm{I}_{\mathrm{a}}$ ) and $\mathrm{Co}^{\mathrm{IV}} / \mathrm{Co}^{\mathrm{III}}$ (peak $\mathrm{II}_{\mathrm{a}}$ ) redox transition assigned to different cobalt oxide species on the electrode surface. ${ }^{26}$ The peaks $\mathrm{I}_{\mathrm{c}}$ and $\mathrm{II}_{\mathrm{c}}$ are cathodic peaks of the reduction of cobalt oxide/hydroxide formed during the anodic cycles that subsequently lead to the production of $\mathrm{Co}$ (II) species according to following sequences:

$$
\begin{aligned}
& \mathrm{Co}(\mathrm{OH})_{2}+\mathrm{OH}^{-} \rightleftarrows \mathrm{CoOOH}+\mathrm{H}_{2} \mathrm{O}+\mathrm{e}^{-} \\
& \mathrm{CoOOH}+\mathrm{OH}^{-} \rightleftarrows \mathrm{CoO}_{2}+\mathrm{H}_{2} \mathrm{O}+\mathrm{e}^{-}
\end{aligned}
$$

Some authors mentioned that an ill-defined anodic peak appeared at a lower potential (peak $\mathrm{III}_{\mathrm{a}}$ ), assigned to the adsorption of oxygen-containing species, $\mathrm{H}_{2} \mathrm{O}$, $\mathrm{OH}^{-}$during consecutive cycles in alkaline solution. ${ }^{30,31}$ Notably, in this work, a drastic increase of the response current is observed with raising the Co:Pt molar ratio. The highest signal current is observed for Co:Pt ratio of 4:1 above which a regression is noticed. On the contrary, the redox peaks of $\mathrm{Co}^{\mathrm{III}} / \mathrm{Co}^{\mathrm{II}}$ and $\mathrm{Co}^{\mathrm{IV}} / \mathrm{Co}^{\mathrm{III}}$ are more ill-defined and the peak currents on $\mathrm{Co} / \mathrm{Pt}$ are smaller than those on CoPtNPs/Pt. The higher peak current on $\mathrm{CoPt}$ (molar ratio of 4:1) electrode is due to an increased roughness of the electro-catalytic film, which leads to a larger exposed surface area in the electrochemical experiments, suggesting better electro-catalytic behavior. It is also seen that CoPt NPs deposited at Co:Pt molar ratio of 4:1 exhibits a much higher adsorption peak $\left(\mathrm{III}_{\mathrm{a}}\right)$, indicating the former have a larger specific active surface area.

The effect of the scan rate $(v)$ on the electrochemical behavior of $\mathrm{Co} / \mathrm{Pt}$ and CoPtNPs/Pt (at various Co:Pt molar ratios) in alkaline solution $(\mathrm{NaOH} 0.1 \mathrm{M})$ is reported in Figure 4a. The peak currents are proportional to the scan rate $(v)$ in the region $\left(10-100 \mathrm{mV} \mathrm{s}^{-1}\right)$, pointing to the electrochemical activity of the surface redox couple. The surface coverage $(\Gamma)$ of $\mathrm{Co}(\mathrm{III})$ species on CoPtNPs/Pt is evaluated using the Laviron's equation. ${ }^{32}$

$I_{\mathrm{p}}=n^{2} F^{2} A \Gamma v(4 R T)^{-1}$

where $A$ is the electrode surface area, $F$ the Faraday constant, $n$ the number of exchanged electrons, $T$ the absolute temperature and $R$ the universal gas constant. Figure $4 \mathrm{~b}$ shows that the surface coverage $\Gamma$ of $\mathrm{Co}(\mathrm{III})$ 

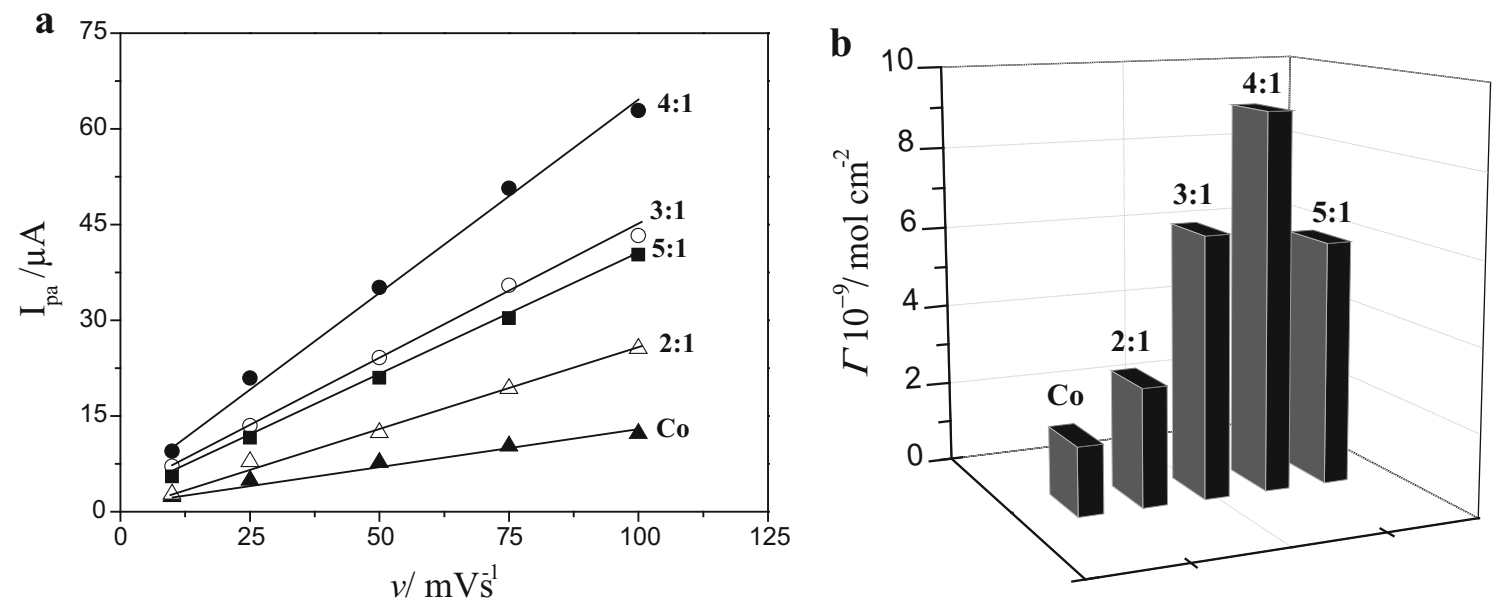

Figure 4. (a) Plots of anodic peak currents vs. the scan rate of CoPtNPs/Pt with different Co:Pt molar ratios. (b) Effects of the Co:Pt molar ratio on surface coverage.

species increases with the Co:Pt ratio. In contrast to the result of Figure 2, it can be concluded that the electroactive $\mathrm{Co}$ (III) deposited on the electrodes increase with increasing the Co:Pt ratio, leading to higher electrocatalytic activity. The lower $\Gamma$ value of the Co/Pt electrode is related to its low electrodeposited yield and different morphology i.e., smaller specific surface area; the highest $\Gamma$ value is found in CoPtNPs/Pt with Co:Pt molar ratio of $4: 1$ and this optimal is selected for further experiments. The decrease in the surface coverage to 5:1 ratio of Co:Pt could be attributed to the enhanced smoothness of the surface morphology of CoPtNPs/Pt electrode.

\subsection{Electrocatalytic oxidation of AA on CoPtNPs/Pt}

The electrocatalytic performance of CoPtNPs/Pt (4:1 molar ratio) for the AA oxidation is examined by cyclic voltammetry. Figure 5 shows the voltammograms at bare $\mathrm{Pt}, \mathrm{Co} / \mathrm{Pt}$ and $\mathrm{CoPtNPs} / \mathrm{Pt}$ in the absence (curve $a, c$ and $e$ ) and in the presence (curve $b, d$ and $f$ ) of AA $(5 \mathrm{mM})$. In the absence of AA, there is no electrochemical response at bare Pt disk electrode (curve a). By contrast, in AA solution a broad, weak and irreversible oxidation peak of AA appears at $0.24 \mathrm{~V}$ (curve $b$ ), suggesting a sluggish electrochemical system. On the contrary, on $\mathrm{Co} / \mathrm{Pt}$ (curve $c$ ), two pairs of peaks corresponding to the reversible $\mathrm{Co}^{\mathrm{III}} / \mathrm{Co}^{\mathrm{II}}$ and $\mathrm{Co}^{\mathrm{VI}} / \mathrm{Co}^{\mathrm{III}}$ redox reaction are observed in blank solution. After addition of AA (curve $d$ ), two anodic peaks at -0.08 (peak $\mathrm{I}_{\mathrm{a}}$ ) and $0.24 \mathrm{~V}$ (peak $\mathrm{II}_{\mathrm{a}}$ ) assigned to the oxidation of AA are observed; the heights of these peaks increase linearly with raising the AA concentration. The anodic peak current related to the oxidation of $\mathrm{Co}$ (II) increases in the presence of AA while the corresponding cathodic

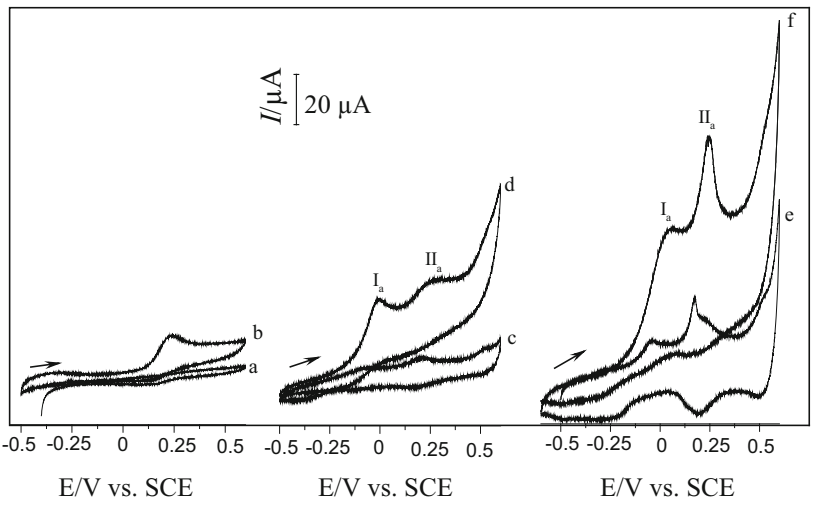

Figure 5. CVs of bare $\mathrm{Pt}(a$ and $b), \mathrm{Co} / \mathrm{Pt}(c$ and $d)$ and CoPt NPs/Pt $(e$ and $f$ ) in $0.1 \mathrm{M} \mathrm{NaOH}$ in the absence $(a, c, e)$ and presence $(b, d, f)$ of AA $(5 \mathrm{mM})$. Scan rate: $50 \mathrm{mV} \mathrm{s}^{-1}$.

currents decrease compared to that in the absence of AA, which is a typical catalytic reaction. In this mechanism, $\mathrm{Co}$ (II) is oxidized to $\mathrm{Co}$ (III), and then $\mathrm{Co}$ (III) oxidizes ascorbic acid followed by the regeneration $\mathrm{Co}$ (II). According to the bi-logarithm dependence of peak currents on the scan rate (data not shown), the electrochemical process occurring at the potential of peak $\mathrm{I}_{\mathrm{a}}$ is complicated by adsorption $\left(\Delta \log I_{\mathrm{p}} / \Delta \log v=0.75\right)$, whereas the electrochemical process at the potential of peak $\mathrm{II}_{\mathrm{a}}$ is complicated by the chemical reaction $\left(\Delta \log I_{\mathrm{p}} / \Delta \log v=0.35\right) .^{33}$

Therefore, the AA oxidation on $\mathrm{Co} / \mathrm{Pt}$ electrode in alkaline medium occurs through redox mediation by electrogenerated $\mathrm{Co}(\mathrm{III})$ in two ways: ${ }^{27}$ the first one involving strong product adsorption in the region -200 to $100 \mathrm{mV}$ (peak $I_{a}$ ), and the other involving soluble forms of both the reactant and product in the region 100 to $350 \mathrm{mV}$ (peak $\mathrm{I}_{\mathrm{a}}$ ). 
The replacement of $\mathrm{Co} / \mathrm{Pt}$ by $\mathrm{CoPtNPs} / \mathrm{Pt}$ changes neither the shape of the voltammogram of AA oxidation (curve $f$ ) nor the peak potentials. Two peaks $\left(E_{\mathrm{p}}=-0.01\right.$ and $\left.0.24 \mathrm{~V}\right)$ correspond to the electrocatalytic oxidation of AA; they are higher than the peaks obtained on the Co/Pt electrode. These enhanced properties are evidently attributed to the high roughness and consequently large active surface area of CoPtNPs/Pt electrode, which produces higher diffusion of AA molecules toward CoPtNPs/Pt and in this way to a better electrocatalytic activity. Comparatively, CoPt$\mathrm{NPs} / \mathrm{Pt}$ is more appropriate for the detection of AA.

Ascorbic acid has two acid protons $\left(\mathrm{H}_{2} \mathrm{~A}\right)$; its oxidation undergoes two successive one electron oxidation steps accompanied by rapid dehydration, which makes the oxidation process irreversible. ${ }^{34,35}$

$$
\begin{aligned}
& \mathrm{AH}_{2} \rightleftarrows \mathrm{AH}^{-}+\mathrm{H}^{+} \\
& \mathrm{AH}^{-} \rightleftarrows \mathrm{A}^{-}+\mathrm{H}^{+}+\mathrm{e}^{-} \\
& \mathrm{A}^{-} \rightarrow \mathrm{DHA}+\mathrm{e}^{-} \\
& \mathrm{DHA}+\mathrm{H}_{2} \mathrm{O} \rightleftarrows \mathrm{P}
\end{aligned}
$$

This mechanism involves a pre-deprotonation process giving ascorbate $\left(\mathrm{AH}^{-}\right)$followed by $1 \mathrm{e}^{-} / 1 \mathrm{H}^{+}$oxidation of the ascorbate to form the radical anion $\left(\mathrm{A}^{-}\right)$, which is further oxidized to dehydroascorbic acid (DHA). The DHA species is rapidly hydrated to form the final product of hydrated bicyclic species (P) as demonstrated by Rueda et al. ${ }^{35}$ and Ruiz et al. ${ }^{36}$

On the basis of the reported results, the following mechanism can be proposed for the mediated oxidation of AA on the modified surface, which is outlined in Figure 6; the redox transition of cobalt species is

$$
\mathrm{Co}(\mathrm{II}) \rightleftarrows \mathrm{Co}(\mathrm{III})+1 \mathrm{e}^{-}
$$

In addition, AA is oxidized on the modified surface via the following reaction:
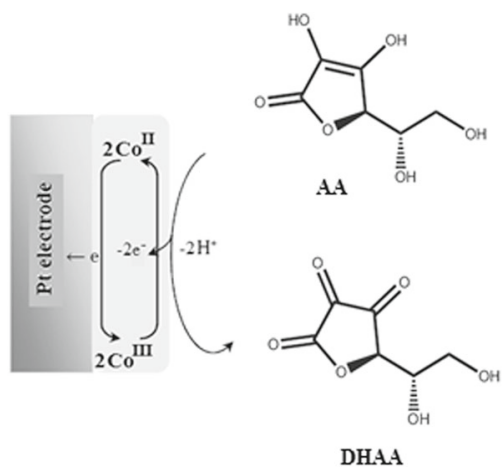

Figure 6. Mechanism for the mediated oxidation of ascorbic acid on the modified surface.
$\mathrm{Co}(\mathrm{III})+\mathrm{AA} \rightarrow$ Intermediate $+\mathrm{Co}(\mathrm{II})$

Where, the intermediate is further oxidized to DHA, through the final electron transfer process:

$\mathrm{Co}(\mathrm{III})+$ Intermediate $\rightarrow \mathrm{DHA}+\mathrm{Co}(\mathrm{II})$

In consequence, $\mathrm{AA}$ is oxidized on $\mathrm{CoPt} / \mathrm{Pt}$ through an irreversible oxidation process, and no obvious reduction peak of AA can be seen in the negative scans of cyclic voltammograms.

The electrocatalytic activity of CoPtNPs film on different substrates, namely, platinum, gold and glassy carbon for AA oxidation was investigated in alkaline medium (data not shown). The results showed that the electrocatalytic activity of CoPtNPs film on different substrates follows the order: CoPtNPs/ Pt $>$ $\mathrm{CoPtNPs} / \mathrm{Au}>\mathrm{CoPtNPs} / \mathrm{GC}$. Thus, the enhanced electrocatalytic activity of the CoPtNPs/Pt electrode is due to the good surface electric conductivity of Pt substrate relative to the surface of $\mathrm{Au}$ and GC electrodes.

\subsection{Chronoamperometric studies}

The potential step chronoamperometry, as well as other electrochemical methods, were also employed for investigating electrochemical processes at chemically modified electrodes. The Chronoamperograms were recorded by setting the working electrode potentials to the desired values and measuring the transient current on the CoPtNPs/Pt surface. Figure 7 shows the chronoamperograms for CoPtNPs/Pt in the absence (curve $a$ ) and presence (curves $c-f$ ) of $\mathrm{AA}$ in the concentration range $(0.1-0.5 \mathrm{mM})$. The applied potential step was $200 \mathrm{mV}$; plotting the net current $v s$. the inverse square roots of

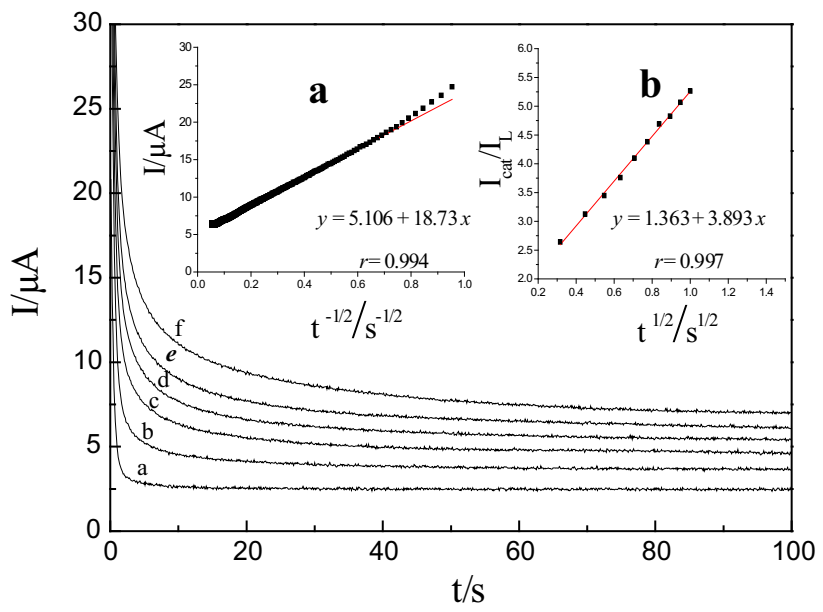

Figure 7. Chronoamperograms of CoPtNPs/Pt in the absence (curve $a$ ) and presence (curves $c-f$ ) of AA in the concentration range $(0.1-0.5 \mathrm{mM})$. Inset (a): the plot of $I_{\text {cat }}$ vs. $t^{-1 / 2}$; Inset (b): the plot of $I_{\text {cat }} / I_{\mathrm{L}}$ vs. $t^{1 / 2}$. 

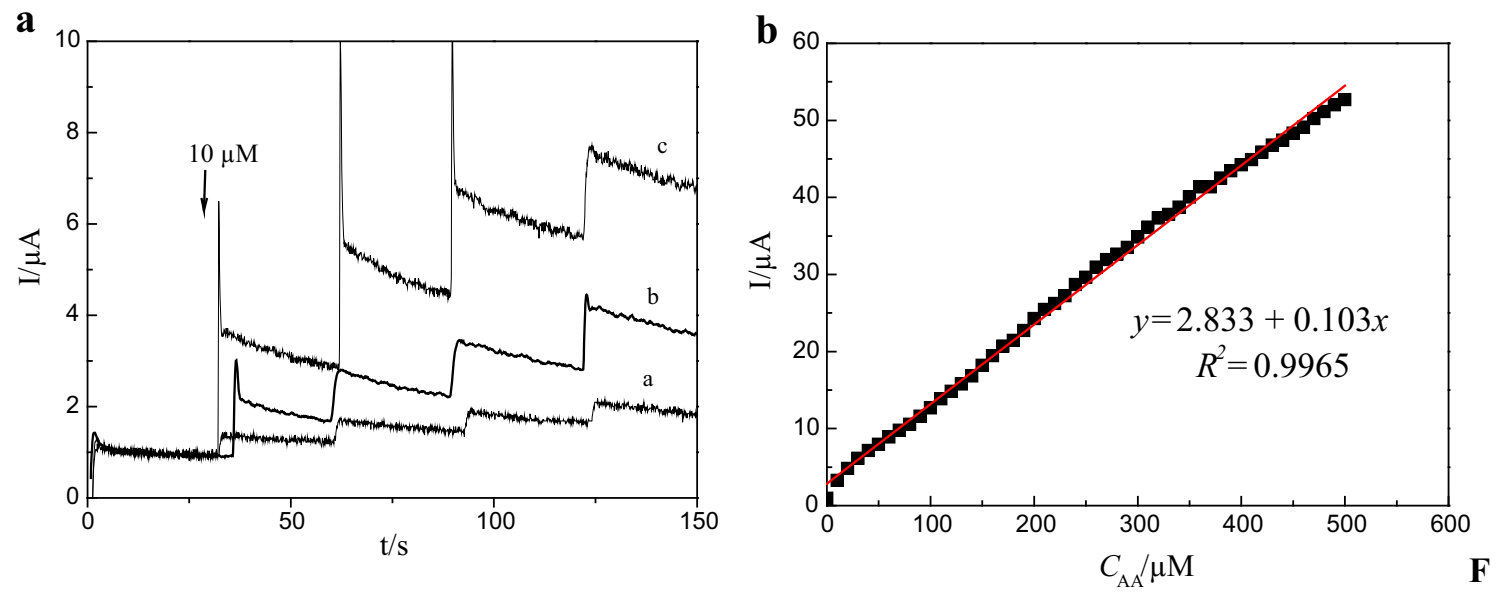

Figure 8. (a) Amperometric responses at bare Pt electrode (curve $a$ ), Co/Pt (curve $b$ ) and CoPt NPs/Pt (curve $c$ ) by successive additions of AA $(10 \mu \mathrm{M})$ in $\mathrm{NaOH}(0.1 \mathrm{M})$ solution. (b) The calibration graph of AA on CoPtNPs/Pt plotted at the working potential $0.20 \mathrm{~V}$.

time shows a linear dependence (Figure 7, inset a). Therefore, a diffusion-controlled process predominates for the AA electrooxidation. The mean the diffusion coefficient $(D)$ of AA can be easily deduced from the slope by using the Cottrell equation: ${ }^{32}$

$I=n F A D^{1 / 2} C_{0}(\pi t)^{-1 / 2}$

where $I$ is the transient current, $C_{\mathrm{o}}$ the bulk concentration and $t$ the elapsed time. The mean diffusion coefficient $D$ for AA is found to be $5.37 \pm 0.05 \times 10^{-5} \mathrm{~cm}^{2} \mathrm{~s}^{-1}$. The chronoamperometry can also be employed to evaluate the catalytic rate constant $(k)$ according to the following relation: ${ }^{37}$

$I_{\text {cat }} / I_{\mathrm{L}}=\gamma^{1 / 2}\left[\pi^{1 / 2} \operatorname{erf}\left(\gamma^{1 / 2}\right)+\exp (-\gamma) / \gamma^{1 / 2}\right]$

where $I_{\text {cat }}$ and $I_{\mathrm{L}}$ are the currents in the presence and absence of AA, respectively, $\gamma=k C_{\mathrm{o}} t$ is the argument of the error function and $t$ is the elapsed time. For $\gamma>$ 1.5, erf $\left(\gamma^{1 / 2}\right)$ is almost equal to unity, and Eq. (14) becomes:

$I_{\text {cat }} / I_{\mathrm{L}}=\gamma^{1 / 2} \pi^{1 / 2}=\pi^{1 / 2}\left(k c_{\mathrm{o}} t\right)^{1 / 2}$

From the slopes of the $I_{\text {cat }} / I_{\mathrm{L}} v s . t^{1 / 2}$ plot (Figure 7, inset b), the mean catalytic rate constant for the electrooxidation reaction is found to be $11.85 \pm 0.12 \times 10^{3} \mathrm{M}^{-1} \mathrm{~s}^{-1}$, two-fold higher than that calculated on $\mathrm{Co} / \mathrm{Pt}$ electrode $\left(6.27 \pm 0.26 \times 10^{3} \mathrm{M}^{-1} \mathrm{~s}^{-1}\right) .{ }^{27}$

\subsection{Amperometric detection of AA on CoPtNPs/Pt}

The amperometry under stirred conditions has a much higher current sensitivity than cyclic voltammetry and is used to estimate the lower limit of detection. Figure $8 \mathrm{a}$ exhibits a typical steady-state catalytic current time response of the CoPtNPs/Pt electrode with successive injections of AA, with an increment of $10 \mu \mathrm{M}$ at a fixed potential of $200 \mathrm{mV}$ in alkaline solution. During the successive addition of AA, a welldefined response is observed, by comparison to both electrodes $\mathrm{Co} / \mathrm{Pt}$ and bare $\mathrm{Pt}$ electrodes, proving stable and efficient catalytic ability of the electrocatalyst $\mathrm{CoPt}$ NPs immobilized onto the Pt electrode surface. This result demonstrated that the CoPtNPs/Pt exhibits a better electrocatalytic activity attributed to its synergistic action since it has a high surface area and an excellent conductivity to promote the electron transfer between AA analyte and bulk-electrode surface. ${ }^{38}$

The calibration plot for CoPtNPs/Pt electrode is given in Figure $8 \mathrm{~b}$. The response current for AA is linear on the concentrations range $(10-500 \mu \mathrm{M})$ with a correlation coefficient $\left(\mathrm{R}^{2}\right)$ of 0.998 . The limit of detection (LOD), defined as the signal-to-noise ratio (3:1), is equal to $0.37 \mu \mathrm{M}$. From these results, one can conclude that the oxidation of AA on the CoPtNPs/Pt electrode can be employed for the determination of AA with good sensitivity over a large concentrations range. Compared with AA sensors given in the literature (Table 1), CoPtNPs/Pt is more sensitive with a larger linearity. Furthermore, the modified electrode is facile to fabricate, relatively inexpensive and mediator-free.

\subsection{Reproducibility, repeatability and stability of CoPt NPs/Pt}

The reproducibility and repeatability of CoPtNPs/Pt were determined. In a series of five different $\mathrm{CoPtNPs} / \mathrm{Pt}$ electrodes elaborated by the same electrochemical technique, a relative standard deviation of $3.2 \%$ was found to 
Table 1. Comparison of the proposed modified electrode with other electrodes for the determination of Ascorbic acid.

\begin{tabular}{lcccc}
\hline Electrode & Sensitivity $\left(\mu \mathrm{A} \mathrm{mM}^{-1}\right)$ & Linear range $(\mu \mathrm{M})$ & LOD $(\mu \mathrm{M})$ & Ref. \\
\hline GCE/MWCNT & 62.5 & $5-120$ & 3.5 & 4 \\
$\mathrm{NiCoO}_{2} / \mathrm{C}$ & 39 & $10-2630$ & 0.5 & 5 \\
{$\left[\mathrm{Ni}(\mathrm{phen})_{2}\right]^{2+} / \mathrm{MWCNTs/GCE}$} & - & $10-630$ & 4 & 6 \\
MWCNT/PNB/GCE & 60.84 & $10-140$ & 2.4 & 7 \\
Chitosan/CPB/GCE & 70.34 & $4-1000$ & 0.8 & 8 \\
CuNPs/c-MWCNT/PANI/Au & 37.5 & $5-600$ & 1 & 9 \\
PoDPoAP & 30.62 & $0.1-10,000$ & 36.4 & 10 \\
Au/HDT/OT & 3.86 & $300-1400$ & 90 & 11 \\
AgNPs/GO/GCE & 43.12 & $10-800$ & 9.6 & 12 \\
Nafion/MWNT/GCE & 8.69 & $80-6000$ & 40 & 13 \\
CoPd/C & 83.71 & $0.1-3420$ & 0.1 & 15 \\
hAu-Ru/GC & 426 & $<5-2000$ & 2.2 & 16 \\
CS/Au-AgNTs/GCE & - & $5-2000$ & 2 & 17 \\
PdNi/C modified GCE & 54 & $10-1800$ & 0.5 & 18 \\
Co/Pt & 74.3 & $10-480$ & 2.5 & 25 \\
CoPtNPs/Pt & 103 & $10-500$ & 0.37 & This work \\
\hline
\end{tabular}

MWCNT/GCE multiwalled carbon nanotubes/glassy carbon electrode, MWCNT/PNB multiwalled carbon nanotubes/poly(Nile blue A), Chitosan/CPB chitosan incorporating cetylpyridine bromide, PoDPoAP poly( $o$-phenylenediamine-co-o-aminophenol), Au/HDT/OT gold/hexanedithiol/1-octanethiol, AgNPs/rGO silver nanoparticle/reduced graphene oxide, hAu-Ru hollow gold/ruthenium, $\left[\mathrm{Ni}(\mathrm{phen})_{2}\right]^{2+}$ nickel(II)-bis(1,10phenanthroline) complex, CS/Au-AgNTs chitosan/gold-silver nanotubes.

Table 2. Determination of ascorbic acid in the vitamin $\mathrm{C}$ tablets using the amperometry method.

\begin{tabular}{lcccc}
\hline Sample & Nominal amount $(\mu \mathrm{M})$ & Found $^{\mathrm{a}}(\mu \mathrm{M})$ & $\mathrm{RSD} \%(\mathrm{n}=5)$ & Recovery $(\%)$ \\
\hline 1 & 50 & 49.5 & 0.90 & 99 \\
2 & 100 & 102 & 0.99 & 102 \\
3 & 250 & 253 & 2.00 & 101 \\
\hline
\end{tabular}

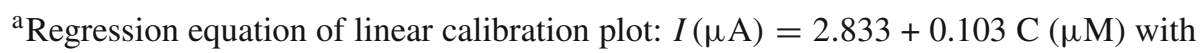
a correlation coefficient of 0.998 .

be $50 \mu \mathrm{MAA}$, showing the reliability of the method. The repeatability was determined by recording five successive amperometric tests for AA concentration of $50 \mu \mathrm{M}$ with the same CoPtNPs/Pt electrode; RSD value of $1.8 \%$ was obtained.

The stability of CoPtNPs/Pt was studied through the response to $50 \mu \mathrm{M}$. When not in use, the electrode was kept in $\mathrm{NaOH}$ solution $(0.1 \mathrm{M})$ at ambient temperature. The data indicate that the current response of the electrode remains almost unchanged after three weeks, suggesting that the CoPtNPs/Pt electrode possesses good long-term stability.

\subsection{Real samples analysis}

To check the applicability and feasibility of the proposed electrochemical sensor, the CoPt NPs/Pt was applied to the AA determination in real pharmaceutical samples with the amperometric method and five replications were performed (Table 2). Good recovery rates for 50,100 and $250 \mu \mathrm{M}$ of ascorbic acid were obtained; proving that the proposed method meets the requirement of analysis and is practical for the determination of ascorbic acid in commercial tablets.

\subsection{Interference study}

The interference of species such as dopamine (DA), uric acid (UA), acetaminophene (AP) and glucose (GL) in the determination of AA was also studied by amperometry. Interfering species were injected with $\mathrm{AA}$ in $\mathrm{NaOH}$ solution under stirring and their response is reported in Figure 9. AA is found to respond in the same way independently of the presence or absence of interferences, thus illustrating that the quantitative determination of AA was in no way affected by interfering species. Further, the response current obtained for these 


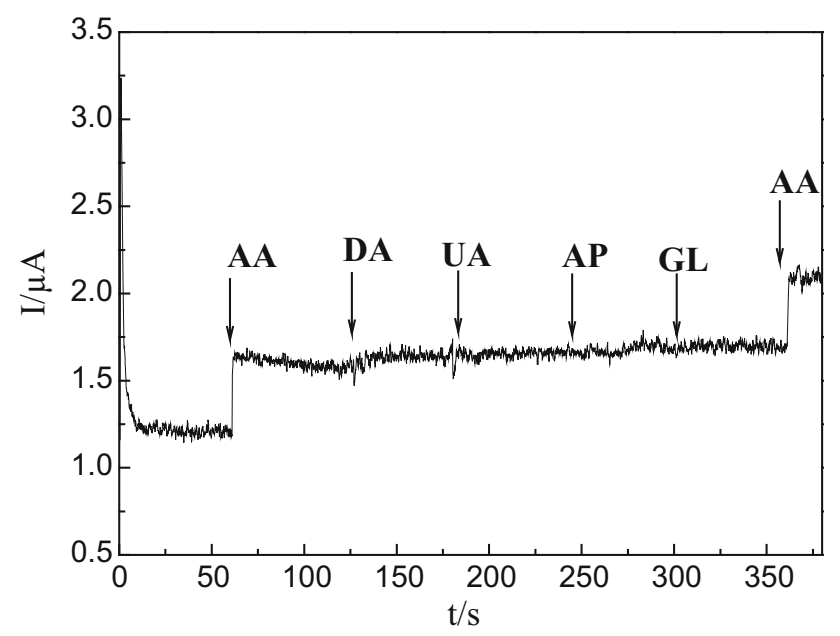

Figure 9. Amperometric responses of CoPtNPs/Pt to AA $(10 \mu \mathrm{M})$ and with the addition of UA $(10 \mu \mathrm{M})$, DA $(10 \mu \mathrm{M})$, $\mathrm{AP}(10 \mu \mathrm{M})$ and GL $(10 \mu \mathrm{M})$ in $0.1 \mathrm{M} \mathrm{NaOH}$.

interfering species was less than $5 \%$ of that observed for AA.

\section{Conclusions}

In this work, bimetallic CoPt nanoparticles were formed on bare Pt electrode by potentiostatic deposition and successfully tested for the electro-oxidation of ascorbic acid in alkaline medium. Comparatively, CoPtNPs/Pt showed a higher catalytic activity and a better stability toward the electrochemical oxidation of ascorbic acid. The electrocatalytic activity of CoPtNPs/Pt has been improved by adjusting the Co:Pt molar ratio in the deposition bath solution. Higher charge-transfer kinetic and better catalytic rate constant were obtained on CoPtNPs/Pt. The final surface concentration of the Co hydroxide species was influenced by the co-existence of platinum. High disorder of the Co hydroxide layer structure was formed on bimetallic CoPtNPs, compared to that on $\mathrm{Co} / \mathrm{Pt}$. More specifically, the electrodes CoPt$\mathrm{NPs} / \mathrm{Pt}$ (molar ratio of 4:1) exhibited the best analytical performance, with a fast response, a linear dependence under optimized conditions over a wide AA concentration $(10-500 \mu \mathrm{M})$ with a low detection limit of $0.37 \mu \mathrm{M}$. Besides, the sensitivity, the repeatability and selectivity of the proposed sensor were satisfying. The results suggest that our proposed CoPtNPs/Pt sensor can be a potential candidate for routine AA analysis.

\section{Acknowledgements}

The authors would like to thank Dr. M. Izeroukkène for his assistance in the SEM characterization. The work is supported financially by the faculty of Sciences (UATL-Laghouat) and the faculty of Chemistry (USTHB-Algiers).

\section{References}

1. Zhang J, Yang H, Shen G, Cheng P, Zhang J and Guo S 2010 Reduction of graphene oxide via L-ascorbic acid Chem. Commun. 461112

2. Begum P and Fugetsu B 2012 Phytotoxicity of multiwalled carbon nanotubes on red spinach (Amaranthus tricolor $\mathrm{L}$.) and the role of ascorbic acid as an antioxidant J. Hazard. Mater. $\mathbf{2 4 3} 212$

3. Fetoui H, Makni M, Mouldi Garoui E and Zeghal N 2010 Toxic effects of lambda-cyhalothrin, a synthetic pyrethroid pesticide, on the rat kidney: involvement of oxidative stress and protective role of ascorbic acid Exp. Toxicol. Pathol. 62593

4. Silva L V, Silva F A S, Kubota L T, Lopes C B, Lima P R, Costa E O, Júnior W P and Goulart M O F 2016 Amperometric sensor based on carbon nanotubes and electropolymerized vanillic acid for simultaneous determination of ascorbic acid, dopamine, and uric acid $J$. Solid State Electrochem. 202389

5. Zhang X, Yu S, He W, Uyama H, Xie Q, Zhang L and Yang F 2014 Electrochemical sensor based on carbonsupported $\mathrm{NiCoO}_{2}$ nanoparticles for selective detection of ascorbic acid Biosens. Bioelectron. 55446

6. Liu X, Li X, Xiong Y, Huang Q, Li X, Dong Y, Liu P and Zhang C 2013 A glassy carbon electrode modified with the nickel(II)-bis (1,10-phenanthroline) complex and multi-walled carbon nanotubes, and its use as a sensor for ascorbic acid Microchim. Acta $\mathbf{1 8 0}$ 1309

7. Kul D, Ghica M E, Pauliukaite R and Brett C M A 2013 A novel amperometric sensor for ascorbic acid based on poly(Nile blue A) and functionalised multi-walled carbon nanotube modified electrodes Talanta 11176

8. Cao X, Xu Y, Luo L, Y Ding and Zhang Y 2010 Simultaneous determination of uric acid and ascorbic acid at the film of chitosan incorporating cetylpyridine bromide modified glassy carbon electrode J. Solid State Electrochem. 14829

9. Chauhan N, Narang J, Rawal R and Pundir C S 2011 A highly sensitive non-enzymatic ascorbate sensor based on copper nanoparticles bound to multi walled carbon nanotubes and polyaniline composite Synth. Met. 161 2427

10. Kong Y, Shan X, Ma J, Chen M and Chen Z 2014 A novel voltammetric sensor for ascorbic acid based on molecularly imprinted poly(o-phenylenediamine-co-oaminophenol Anal. Chim. Acta 80954

11. Raoof J B, Kiani A, Ojani R, Valiollahi R and RashidNadimi S 2010 Simultaneous voltammetric determination of ascorbic acid and dopamine at the surface of electrodes modified with self-assembled gold nanoparticle films J. Solid State Electrochem. 141171

12. Kaura B, Pandiyan T, Satpatic B and Srivastava R 2013 Colloids Surf. B 11197

13. Li G, Yang S, Qu L, Yang R and Li J 2011 Simultaneous voltammetric determination of uric Acid and ascorbic acid using a carbon paste electrode modified with multiwalled carbon nanotubes/nafion and cobalt(II) nitrosalophen J. Solid State Electrochem. 15161

14. Prieto F, Coles B A and Compton R G 1998 Mechanistic determination using arrays of variable-sized channel 
microband electrodes: the oxidation of ascorbic acid in aqueous solution J. Phys. Chem. B 1027442

15. Yang F, Wang J, Cao Y, Zhang L and Zhang X 2014 A highly sensitive ascorbic acid sensor based on carbonsupported CoPd nanoparticles Sensor Actuat. B-Chem. 20520

16. Jo A, Kang M, Cha A, Jang H S, Shim J H, Lee NS, Kim M H, Lee Y and Lee C 2014 Nonenzymatic amperometric sensor for ascorbic acid based on hollow gold/ruthenium nanoshells Anal. Chim. Acta 81994

17. Yang P, Gao X, Wang L, Wu Q, Chen Z and Lin X 2014 Amperometric sensor for ascorbic acid based on a glassy carbon electrode modified with gold-silver bimetallic nanotubes in a chitosan matrix Microchim. Acta $\mathbf{1 8 1}$ 231

18. Aparna T K and Sivasubramanian R $2018 \mathrm{NiFe}_{2} \mathrm{O}_{4}$ nanoparticles-decorated activated carbon nanocomposite based electrochemical sensor for selective detection of dopamine in presence of uric acid and ascorbic acid J. Chem. Sci. 13014

19. Zhang X, Cao Y, Yu S, Yang F and Xi P 2013 An electrochemical biosensor for ascorbic acid based on carbon-supported PdNi nanoparticles Biosens. Bioelectron. 44183

20. Hamidi-Asl E, Raoof J B, Naghizadeh N, Sharifi S and Hejzib M S 2015 A bimetallic nanocomposite electrode for direct and rapid biosensing of p53 DNA plasmid $J$. Chem. Sci. 1271607

21. Tsai T H, Thiagarajan S and Chen S M 2010 Green synthesized $\mathrm{Au}-\mathrm{Ag}$ bimetallic nanoparticles modified electrodes for the amperometric detection of hydrogen peroxide J. Appl. Electrochem. 402071

22. Jeena S E and Selvara J U 2016 Facile growth of Ag@Pt bimetallic nanorods on electrochemically reduced graphene oxide for an enhanced electrooxidation of hydrazine J. Chem. Sci. 128357

23. Guan H, Chao C, Lu Y, Shang H, Zhao Y, Yuan S and Zhang B 2016 PtNi nanoparticles embedded in porous silica microspheres as highly active catalysts for $p$ nitrophenol hydrogenation to $p$-aminophenol J. Chem. Sci. 1281355

24. Jiang S, Ma Y, Jian G, Tao H, Wang X and Fan Y 2009 Facile construction of Pt-Co/CNx nanotube electrocatalysts and their application to the oxygen reduction reaction Adv. Mater. 214953

25. Kakade B A, Tamaki T, Ohashi H and Yamaguchi T 2012 Highly active bimetallic PdPt and CoPt nanocrystals for methanol electro-oxidation J. Phys. Chem. C 116 7464
26. Chen Q-S, Sun S-G, Zhou Z-Y, Chen Y-X and Deng S-B 2008 CoPt nanoparticles and their catalytic properties in electrooxidation of $\mathrm{CO}$ and $\mathrm{CH}(3) \mathrm{OH}$ studied by in situ FTIRS Phys. Chem. 103645

27. Doulache M, Saidat B and Trari M 2017 Electrocatalytic performance of cobalt microparticles film-modified platinum disk electrode for amperometric detection of ascorbic acid J. Anal. Chem. 72333

28. Ghartavol H M, Moakhar R S and Dolati A 2017 Electrochemical investigation of electrodeposited platinum nanoparticles on multi walled carbon nanotubes for methanol electro-oxidation J. Chem. Sci. 1291399

29. Cheng Y, Yu G, Tang L, Zhou Y and Zhang G 2011 Selfassembled dendritic nanowires of Au-Pt alloy through electrodeposition from solution under AC fields J. Cryst. Growth 334181

30. Song Y, He Z, Zhu H, Hou H and Wang L 2011 Electrochemical and electrocatalytic properties of cobalt nanoparticles deposited on graphene modified glassy carbon electrode: Application to some amino acids detection Electrochim. Acta $\mathbf{5 8} 757$

31. Bruckenstein S and Shay M 1985 An in situ weighing study of the mechanism for the formation of the adsorbed oxygen monolayer at a gold electrode J. Electroanal. Chem. 188131

32. Bard A J and Faulkner L R 2001 Electrochemical methods (New York: Wiley)

33. Bard A J and Faulkner L R 1980 Electrochemical methods (New York: Wiley)

34. Lu X, Jin J, Kang J, Lv B, Liu H and Geng Z 2003 The characterization of 5- ([4-(4-mercapto)- phenylmethoxy] phenyl-10,15,20-tris(phenyl) por-phyrin cobalt(II) selfassembled monolayers (SAMs) and its electrocatalytic oxidation for ascorbic acid Mater. Chem. Phys. 77952

35. Rueda M, Aldaz A and Sanchez-Burgos F 1978 Oxidation of L-ascorbic acid on a gold electrode Electrochim. Acta 23419

36. Ruiz J J, Aldaz A and Dominguez M 1978 Mechanism of L-ascorbic acid oxidation on a mercury electrode. II. Basic medium Can. J. Chem. 561533

37. Kutner W, Wang J, Lher M and Buck R P 1998 Analytical aspects of chemically modified electrodes: classification, critical evaluation and recommendations Pure Appl. Chem. 701301

38. Rao D, Sheng Q and Zheng J 2016 Novel nanocomposite of chitosan-protected platinum nanoparticles immobilized on nickel hydroxide: facile synthesis and application as glucose electrochemical sensor J. Chem. Sci. 128 1367 